\title{
Determinants of Neonatal Mortality in the Health Districts of Labe, Mamou, Faranah and Kankan
}

\author{
Diallo Ibrahima Sory ${ }^{1}$, Diallo Sory ${ }^{1}$, Camara Yero Boye ${ }^{2}$, Diallo Thierno Saïdou ${ }^{3}$, Conte N'faly ${ }^{1}$, \\ Koulibaly Mamadou Korka ${ }^{1}$, Cisse Moussa ${ }^{1}$, Kaba Djèney Fadima ${ }^{2}$ Daffe Mamadi $^{4}$ \\ ${ }^{1}$ Department of Neonatology, Institute of Child Nutrition and Health, Conakry, Republic of Guinea \\ ${ }^{2}$ Strategy and Development Office, Ministry of Health, Conakry, Republic of Guinea \\ ${ }^{3}$ National HIV Programme, Ministry of Health, Conakry, Republic of Guinea \\ ${ }^{4}$ Food and Nutrition Division, Ministry of Health, Conakry, Republic of Guinea
}

Email address:

diallopediatrie@yahoo.fr(D. I. Sory)

\section{To cite this article:}

Diallo Ibrahima Sory, Diallo Sory, Camara Yero Boye, Diallo Thierno Saïdou, Conte N'faly, Koulibaly Mamadou Korka, Cisse Moussa, Kaba Djèney Fadima, Daffe Mamadi. Determinants of Neonatal Mortality in the Health Districts of Labe, Mamou, Faranah and Kankan. Central African Journal of Public Health. Vol. 5, No. 6, 2019, pp. 246-251. doi: 10.11648/j.cajph.20190506.13

Received: September 5, 2019; Accepted: September 24, 2019; Published: October 10, 2019

\begin{abstract}
Neonatal mortality is still a real public health problem in Guinea. Indeed, despite the remarkable progress made in the country, neonatal mortality is still high: 20 per 1000 live births (1). Regional disparities are significant, with the regions of Mamou (32\%o), Faranah (28\%), Labé (26\%) and Kankan (26\%) being the most affected. Objective: To determine the main factors related to neonatal deaths in the Faranah, Mamou, Labé and Kankan regions. This is a descriptive qualitative study that examines the perspectives of health service users, local decision-makers and providers on the causes of neonatal mortality in the Labé, Mamou, Faranah and Kankan regions. The main direct medical causes of neonatal mortality cited by participants were: prematurity, neonatal infections, low birth weight, hypoglycemia, hemorrhagic diseases of the newborn. This workshop concluded that neonatal mortality depends on a multitude of economic, political, socio-cultural and health factors, all of which endanger the lives of newborns.
\end{abstract}

Keywords: Neonatal Mortality, Determinants, Health District

\section{Introduction}

According to the 2014 population census, Guinea's population is estimated at $11,883,516$ in 2018 [1]. More than half $(51 \%)$ are under 18 years of age. Adolescents represent more than $23 \%$ of the population, and most of them live in rural areas. Gender inequalities and disparities remain widespread in access to basic social services and economic opportunities. With a total fertility rate of 5.1 children per woman, Guinea is one of the countries in the region with high population growth, with a natural growth rate of $2.3 \%$ per year.

Neonatal mortality is still a real public health problem in Guinea. Indeed, despite the remarkable progress made in the country, neonatal mortality is still high: 20 per 1000 live births [2]. Regional disparities are significant, with the regions of Mamou (32\%), Faranah (28\%), Labé (26\%) and
Kankan (26\%) being the most affected (See figure 1, image in blue).

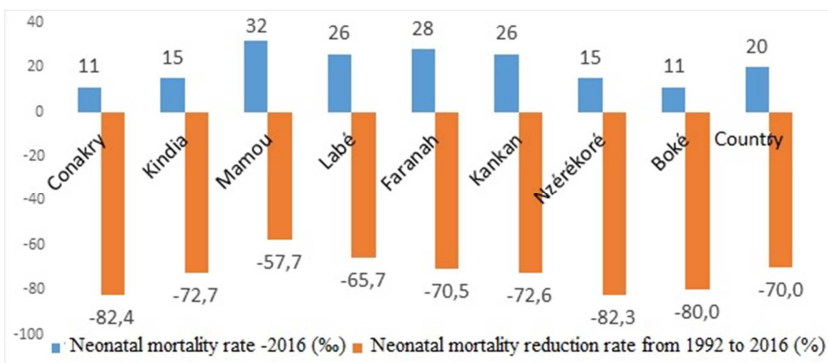

Figure 1. Neonatal mortality rate (2016) and its reduction (1992-2016).

An examination of the evolution of neonatal mortality shows the extent of progress in this area. Indeed, over the past 24 years (1992 to 2016), neonatal mortality has risen from 66.6 deaths per 1000 live births to its current level 
(20\%) for the country [2]. This improvement is noticeable in all administrative regions. But the rate of reduction is not uniform in all regions. Again, the regions of Mamou, Labé, Faranah and Kankan are those where neonatal mortality is declining more slowly (see Figure 1, orange image).

This graph clearly shows that the rate of reduction in neonatal mortality is much lower in Mamou, Labé, Faranah and Kankan than in the other regions since 1992, with the situation being more marked in Mamou and Labé.

This raises the following question: Why is neonatal mortality higher in these regions than in others?

Answering this question would require a comparison of neonatal mortality factors in these regions with those in other regions.

This study is the first in a series that will be organized to try to answer this question. It therefore focused on identifying the causes of neonatal mortality in the regions of Mamou, Labé, Faranah and Kankan. Indeed, the studies carried out in this field in the country are of the demographic and health survey type. None of them were interested in the causes.

It must be complemented by comparative studies with other regions to identify the specific factors that explain the highest neonatal mortality and in these regions.

The results can be used to formulate hypotheses for further studies. They can also be used to plan specific maternal and neonatal mortality reduction interventions targeting these regions.

\subsection{General Objective}

Determine the main factors related to neonatal deaths in the Faranah, Mamou, Labé and Kankan regions.

\subsection{Specific Objectives}

Identify neonatal death factors related to the health system Determine environment-related neonatal death factors Identify socio-cultural factors of neonatal death Identify the causes of neonatal deaths

\section{Materiel and Methods}

This is a descriptive qualitative study that examines the perspectives of health service users, local decision-makers and providers on the causes of neonatal mortality in the Labé, Mamou, Faranah and Kankan regions.

\subsection{Study Setting}

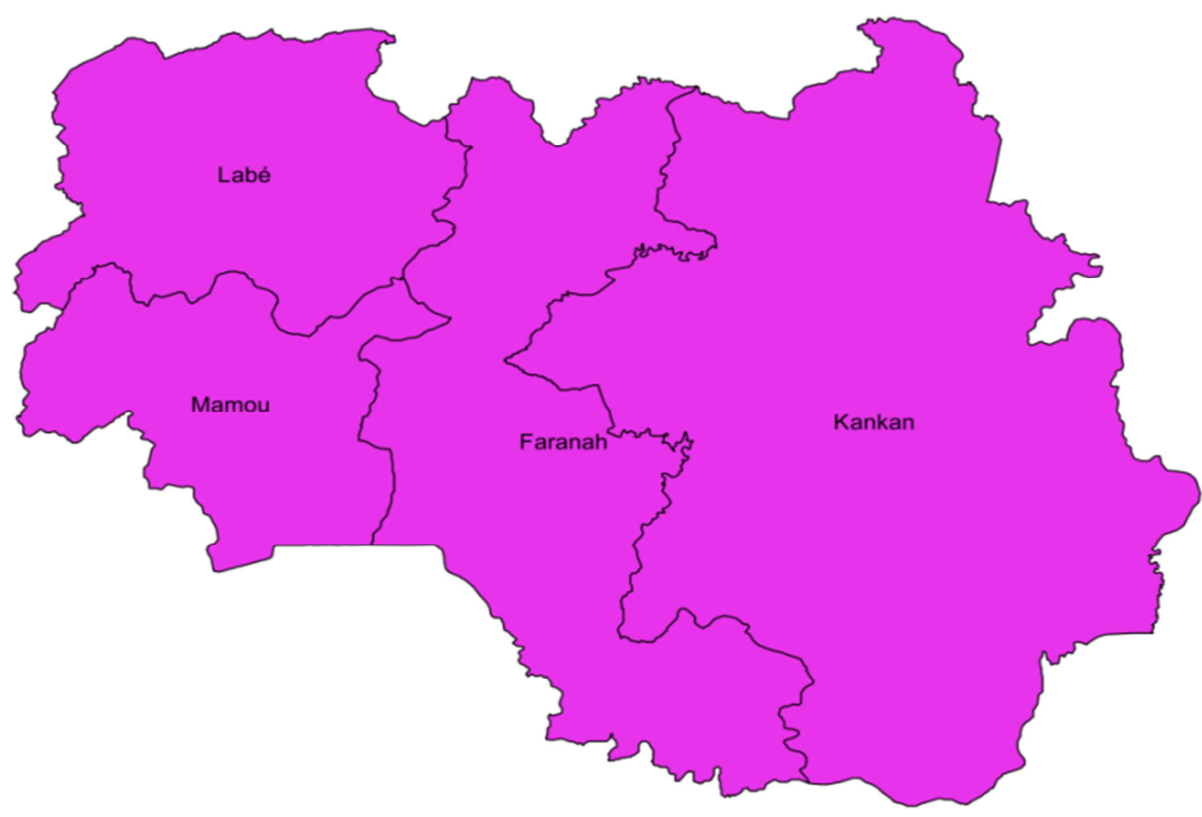

Figure 2. Map of the administrative regions of the study setting.

As mentioned above, the study took place in the administrative regions of Mamou, Labé, Kankan and Faranah.

\subsubsection{Administrative Region Mamou}

The district's relief is characterized by mountains and plateaus varying between $700 \mathrm{~m}$ and more than $1000 \mathrm{~m}$ in altitude, severely limiting the possibilities of grouping the population into large urban areas. Thus, the population is rather scattered in small hamlets far from each other and connected by tracks that snake the mountains. These tracks are difficult to use or even not by vehicle, even by motorcycle. As a result, populations, especially children and pregnant women, have limited access to health services, even in emergencies. In terms of human resources, as of 31 December 2017, the region had a total of 934 health workers (doctors, midwives, state nurses and technical health workers "THW"), with THW representing 39\% of this workforce. Thus, the density of human resources is 1.2 care workers per 1000 inhabitants [3]. 


\subsubsection{Administrative Region Faranah}

The region is a transition zone between the Fouta Djallon mountains and the plateaus of western Upper Guinea. The region's plateaus are generally lateritic, at altitudes ranging from 200 to 400 metres, cut by numerous rivers: Niger, Tinkisso, Mafou, Niandan, Kouya, Bafing and Banié, leading to the isolation of some villages during flood periods with the consequent reduction in access to healthcare facilities [1]. The Faranah region includes 4 prefectures which are: Dabola, Dinguiraye, Faranah and Kissidougou subdivided into 42 communities. In terms of health, it should be noted that $100 \%$ of the Complete Obstetrico-Neonatal Emergency Care (CONUC) facilities are available while only $22 \%$ of the Basic Obstetrico-Neonatal Emergency Care (BONEC) facilities are satisfied.

Faranah has 1024 care workers, as defined in Mamou, with 43.1\% THW and a Human Resources (HR) density of 1.0 care workers per 1000 inhabitants [3]

\subsubsection{Administrative Region Labé}

It covers a huge area of bowls and is home to a large number of rivers and springs. Thus, the nature of the relief and the hydrographic network make access conditions to the various localities in the region quite restrictive. Here too, as in Mamou, populations are scattered in remote hamlets with difficult access, a sign of difficulties in accessing health services for the population. Fertility is high (4.9 children per woman on average) and early (45.8\% of adolescent girls are mothers) [3]. All emergency obstetric and neonatal care facilities are functional compared to $44.4 \%$ of SONUB.

The total number of caregivers (doctor, midwife, state nurse and technical health worker) is 976 care workers, $34.6 \%$ of whom are ATS. The density of HR is 0.9 caregivers per 1000 in habitants [3].

\subsubsection{Administrative Region Kankan}

It covers an area of $72,145 \mathrm{~km}^{2}$ with an estimated population of 2,218,705 inhabitants in 2018, or an average density of 31 inhabitants per $\mathrm{km}^{2}$. The region has five (5) prefectures: Kankan, Kérouané, Kouroussa, Mandiana and Siguiri. The region faces enormous challenges to its development, including: isolation of production areas, environmental degradation, low household income, inadequate health infrastructure and poor management of the resources mobilized [1].

\subsection{The Targets of the Survey}

Health service users:

i. women's groups (7),

ii. youth associations (8),

iii. religious associations (7),

iv. traditional birth attendants (6),

v. mothers (7),

vi. COSAH members (8).

Decision-makers:

i. Prefectural health directors (8),

ii. Regional health directors (2), iii. Mayors and municipal councilors (12).

Health personnel in health care and administrative services:

i. Gynecologists (7),

ii. Pediatricians (7),

iii. Midwives (8),

iv. THW (7),

v. State nurses (7),

vi. General physicians (7).

A total of 108 participants took part in this workshop.

\subsection{Inclusion Criteria}

Belong to one of the study's target groups and consent to participate in the study.

\subsection{Non-inclusion Criterion}

Two criteria are used to exclude a person from the survey

1. Do not match any of the defined targets.

2. Not be willing to participate in the survey

Data collection: Two teams of investigators visited the field: one in Mamou and Labé from 16 to 19/11/2018 and the second from 15 to 20/11/2018 in Faranah and Kankan. In accordance with UNICEF instructions, a regional reflection workshop was organized in each of the 4 health districts. In the introductory plenary session, the issue of neonatal mortality, motivation, objectives and usefulness of the survey were explained to participants. Participants were then divided into three groups: users, decision-makers and providers. The terms of reference of the working groups were presented and clarified by the facilitators. The consensus results of each group's discussions were reported and adopted in plenary. The major limitation of this data collection approach is that the causes or factors of neonatal or maternal death that have not been agreed upon are not considered as such, although they could play a significant role in some settings.

\section{Results and Comments}

All groups of participants (users, decision-makers, providers) in the workshops on neonatal mortality organized in all 4 regions concerned stated that the low qualification of providers, their numerical inadequacy, the inadequacy of care structures, the distance of care structures from the population's habitat, the insufficient quality of services, the poor state of roads, poverty and population ignorance... are the main factors explaining neonatal mortality. These findings are likely to be of greater importance in rural areas than in urban areas. We therefore investigated whether there is any data that supports these statements. We particularly examined care structures, human resources (their quantity, quality and distribution), and survey data.

\subsection{Mortality Factors Related to the Health System}

Available infrastructure data (see Table 1) show that in these regions, CONEC services are satisfied everywhere $(100 \%)$ except in Kankan. On the other hand, the structures 
offering BONEC services are clearly insufficient. Indeed, the level of satisfaction of the needs in these services varies from $22 \%$ in Kankan to $44 \%$ in Labé.
The situation of Obstetric and Neonatal Emergency Care (ONEC) services in the 4 regions covered by this study is summarized in Tables 1.

Table 1. Distribution of ONEC by type and region.

\begin{tabular}{|c|c|c|c|c|c|c|c|c|c|c|}
\hline \multirow{2}{*}{ Region } & \multirow{2}{*}{ Population 2017} & \multicolumn{3}{|c|}{ ONEC existant } & \multicolumn{3}{|c|}{ ONEC requis } & \multicolumn{3}{|c|}{ Gap } \\
\hline & & B & $\mathbf{C}$ & total & B & $\mathrm{C}$ & Total & B & $\mathbf{C}$ & Total \\
\hline Faranah & 1035162 & 2 & 2 & 4 & 8 & 2 & 10 & 6 & 0 & 6 \\
\hline Kankan & 2157381 & 6 & 4 & 10 & 17 & 5 & 22 & 11 & 1 & 12 \\
\hline Mamou & 803483 & 3 & 2 & 5 & 6 & 2 & 8 & 3 & 0 & 3 \\
\hline Total & 5087659 & 15 & 11 & 26 & 40 & 12 & 51 & 25 & 1 & 26 \\
\hline
\end{tabular}

Source: Ministry of Health [4]

Although we did not have data to compare rural and urban areas, it is possible, if not likely, that this situation is more critical in rural areas. Three years earlier the situation was worse (no SONUB structure and very few CONEC in the country and therefore in these 4 regions) [5]. These data are consistent with the "scarcity, remoteness, difficult access to structures" reported by participants. Rural areas where neonatal mortality is higher may be more affected. The human resource density (Table 2) in these regions is lower (Kankan and Faranah) or equal (Labé and Mamou) to the national average (0.8 agents per 1000 inhabitants). This density is calculated by dividing the sum of the number of doctors, midwives, state nurses and ATSs (technical health workers) in each region by its population. One might therefore think that human resources are not a particular problem. However, an examination of the composition of health care teams shows that THW constitute between $40 \%$ (in Mamou) and 57\% (in Kankan) of the staff. However, ATS are the least qualified professional category.

Table 2. Distribution of Staff by region as at 31 December 2017.

\begin{tabular}{lllll}
\hline Professional Corps & Kankan & Labé & Mamou & Faranah \\
\hline Technical Health Worker & 761 & 390 & 267 & 336 \\
Density & 0,4 & 0,4 & 0,3 & 0,3 \\
Médecin & 231 & 160 & 138 & 166 \\
Density & 0,1 & 0,1 & 0,2 & 0,2 \\
State nurse & 248 & 248 & 184 & 190 \\
Density & 0,1 & 0,2 & 0,2 & 0,3 \\
Midwife & 106 & 87 & 80 & 81 \\
Density & 0,05 & 0,08 & 0,10 & 0,2 \\
Total & 1346 & 885 & 669 & 0,08 \\
Population & 2157381 & 1091633 & 803483 & 773 \\
Density & 0,6 & 0,8 & 0,8 & 0,2 \\
\hline
\end{tabular}

Source: Ministry of Health [4]

If these indicators were disaggregated by residential area of the population, they could be more pronounced in rural areas. Indeed, the latest human resources analysis shows that in $2012,86 \%$ of the staff working in rural areas was THW [3]. Despite the improvements over the past three years, there is no evidence that the order of magnitude has reversed. In addition, these staff in rural areas receive little or no continuing training, supervision or technical support, which contributes to their lack of motivation. It could therefore be argued that the problem of human resources, particularly in rural areas, arises in terms of the quality of existing human resources. These data suggest that participants in the think tanks may be right when they report "low levels of claimants" or "low qualifications of claimants". They are also consistent with higher neonatal mortality in rural areas.

It should also be noted that services, infrastructures, personnel and, above all, their poor financial and geographical accessibility, which most often lead to delays in the decision-making process, in the provision of health services and in the provision of care. In the study, by $\mathrm{P}$.
Sauvegrain et al, many respondents also mentioned the problem of the organization of care, the quality of care, the lack of staff and practitioners, and the deterioration of working conditions as poor indicators of neonatal mortality [5].

Environmental neonatal mortality factors

The different statements made by participants from the four Health Districts on neonatal mortality factors related to the environment are: worsening degradation of roads and bridges, ecosystem, isolation of housing areas, insalubrity, lack of decent housing, pollution, and the effects of climate change, distance of the population from health facilities. On the other hand, a study of the impact of distance from home to the nearest maternity hospital on neonatal mortality and stillbirths has not found a national link, although in more rural areas too long a distance can be harmful to the health of newborns [6]. Paradoxically, it has been found in France that neonatal mortality is higher for women whose homes are close to maternity [7].

The delay in accessing health facilities is exacerbated by 
the poor condition of roads, especially during wintering when they are cut off by river water, marigolds and rivers. Indeed, it is relatively difficult to get to a health center if the roads leading to it are not passable. As a result, the environment is one of the main causes of child deaths, especially in the neonatal period. According to WHO, worldwide, newborns are more exposed than anyone else to a myriad of environmental threats [8].

\subsection{Neonatal Mortality Linked to Socio-cultural Factors}

In all four regions, participants' statements highlighted the existence of socio-cultural factors that influence or explain the adoption of behaviors that promote neonatal deaths. These include, among others, women's illiteracy, poverty, under-utilization of family planning, use of tradi-pratciens, false rumors, female genital mutilation, gender-based violence, influences of customs and morals, taboos and prohibitions. In these regions, the period of pregnancy is often surrounded by prejudice. On this occasion, some women are subjected to food bans and taboos that deprive them of the protein-rich diet and other nutrients necessary for the development of the fetus. However, the proper development of the fetus depends on the functioning of the fetal-placental unit and, in particular, on the nutritional intakes that contribute to the growth and storage of different nutrients [9]. The use of traditional Pratciens exposes the lives of newborns to the risk of death. The under-utilisation of health services, for example, is a consequence of the general level of understanding among women who ignore the signs of severity of obstetric and neonatal emergencies, the importance of family planning, the danger of early marriage and of excision due to their illiteracy. The predominance of illiterates reflects the structure of Guinean society. According to MICS 2016, only less than two in five women (39\%) can read and write [10]. This rate varies considerably according to socio-demographic characteristics; its value in urban areas is three times higher than in rural areas $(59 \%$ in urban areas compared to nearly $18 \%$ in rural areas). The benefits of literacy, especially for women, are well known; greater labor market participation, less early marriages, improved health and nutritional status of children and their families, all factors that contribute to reducing poverty and improving future prospects. There is a strong association between women's level of education or literacy and the use of reproductive, maternal and child health services. Maternal and neonatal mortality levels are significantly higher in countries with high female illiteracy rates [11].

The study conducted by Ngayawanaka in Cameroon in 2012 showed that the mother's level of education reduced the risk of neonatal death in the Great South in 1991 and in the Great West in 2004 [12]. False rumors also reduce public confidence in health facilities.

According to Akoto (1985), cultural traditions, norms and practices influence neonatal mortality through the behavior, attitude and beliefs they induce in individuals [13]. Sociocultural factors are mainly responsible for the delay in making decisions about health care use. It is therefore important for providers and policy makers to be aware of these factors that are mentioned above. Above all, the influence of religious leaders, healers or traditional birth attendants on the attitude of the community should not be underestimated. For example, pregnant women may choose to give birth in dangerous conditions for cultural and religious reasons such as refusal to be seen by a man or the greatest trust in traditional Pratciens, marabouts and others. Participants also identified other socio-cultural factors such as: early marriage, ignorance of community members and home delivery. The MICS 2016 results indicate that in Guinea $42 \%$ of births in the two years preceding the survey took place at home [10]. It is in rural areas that home births are more frequent $(56 \%)$ than in urban areas $(14 \%)$. The regions of Mamou (74\%), Labé (64\%) and Faranah 64\% have the highest proportions of women giving birth at home. Home births are more frequent among women with no education $(50 \%)$ and those living in very poor households (76\%). According to the study conducted in Bakel, Senegal, 11 out of 16 women who died $(68 \%)$ gave birth at home compared to only $4(25 \%)$ in health facilities [14]. Home childbirth, which is a customary practice, is a danger according to $\mathrm{WHO}$, leading to different risks for the child and the mother (maternal death, neonatal trauma, stillbirth, fistula) [15]. The persistence of this practice is explained by the presence of traditional birth attendants in communities and families. These midwives enjoy a certain reputation built on several years of experience and expertise, the humanism they demonstrate unlike midwives, but also the socio-cultural burdens that promote the maintenance of home delivery in the community.

Causes of neonatal mortality

The direct causes of neonatal mortality cited by participants from the four regions were: prematurity, neonatal infections, low birth weight, the anoxia, hypoglycemia, hemorrhagic diseases of the newborn. In the reference neonatology unit of the Gabriel Touré University Hospital (Mali), prematurity, perinatal anoxia and neonatal infection accounted for $80.5 \%$ of hospitalization causes over the five years (2008-2012), with percentages of $30.2 \%, 27.4 \%$ and $22.9 \%$ respectively [16]. The factors such as "'lack of awareness of the importance of attending health facilities, lack of adequate infrastructure, incompetence of some health workers, lack of resuscitation services in hospitals, delayed admission to health services, insufficient resuscitation equipment for newborns, poverty, lack of awareness among users, distance from health centers and lack of transport" were also mentioned by the participants. In the Democratic Republic of Congo, Kataméa $T$ et al, found that parents made little use of public transport $(26.7 \%)$ and in the majority of cases transport was done on foot, but under no circumstances was the ambulance used. According to them, the majority of outlying maternity hospitals do not have specialized means of transport for the transfer of newborns; hence newborns are transferred to the hands of parents on foot, exposing them to hypothermia and hypoglycemia because they do not benefit from heat blankets or any treatment along the way to health 
services [17].

The distance from the peripheral centers of the maternity centers of reference and the transport in poor conditions lead to a risk of aggravation of the newborn's condition. There is currently no doubt that it is possible to significantly reduce perinatal mortality and morbidity rates by organizing the identification of "mother-child" couples at risk, to refer them antenatal to centers better equipped with techniques and human resources to care for them. It emerges from these different statements that neonatal mortality may depend on several factors that may be related to the mother, and the child, such as Socio-economic, cultural or contextual, environmental, behavioral, health and other factors. The current interdependence between the social, health and cultural spheres is undeniable, making the global approach to neonatal mortality a necessity.

\section{Conclusion}

The participants' various statements show that the factors contributing to neonatal mortality are at the community level (ignorance, illiteracy, poverty, traditional treatment, beliefs and taboos, home delivery,), at the environmental level (isolation, poor road conditions, lack of logistics, lack of communication), at the health system level (lack of competent personnel, delayed intervention, low level of providers, poor quality of care, poor quality of reception, lack of health infrastructure and facilities.), at the medical level (hemorrhagic diseases of the newborn, anemia, prematurity, neonatal infections and low birth weight, etc.) in the newborn.

Neonatal mortality therefore depends on a multitude of economic, political, socio-cultural and health factors, all of which endanger the lives of newborns. From this point of view, any policy must go beyond the framework of a vertical programmer and must be part of a more global multisector and multidimensional policy framework integrating all actors in society without discrimination and taking into account all these factors contributing to neonatal deaths.

\section{References}

[1] Ministry of Planning and International Cooperation. Third general population and housing census. RGPH3, 2014.

[2] Ministry of Planning and International Cooperation. Demographic and Health Survey 2016.
[3] Ministry of Health, Human Resources for Health Situation Analysis, July 2012.

[4] Ministry of Health, SONU Evaluation, 2017.

[5] P. Sauvegrain, R. Rico-berrocal, J. Zeitlin. What are the causes of high perinatal and infant mortality in Seine-Saint-Denis? Consultation of the department's professionals through a Delphi process. J Gynecol obstetr Biol Reprod 2016.

[6] Pilkington H, Blondel B, Papiernik E, Cuttini M, Charreire H, Maier RF, et al. Distribution of maternity service unit and risk of fetal and neonatal mortality in France. Eur J Publ Health 2014.

[7] Combier E, Charreire H, Le Vaillant H, Michaut F, Ferdynus C, Amat-Rose JM, et al Perinatal anequalities and accessibility of maternity service in a rural French region: closing maternity units in Burgundy. Health Place 2013; 24: 225-3.

[8] World Health Organization, A Healthy Environment for Healthy Children: Key Messages for Concrete Action, 2010.

[9] Patricia Fischer-Ghanassia, E. Ghanassia. EndocrinologyNutrition. Vernazobres-Grego 6th Edition (2012), p 458-459. ISBN: 978-2-8183-0367-2.

[10] National Institute of Statistics. Multiple Indicator Cluster Survey (MICS, 2016), Final Report, Conakry, Guinea.

[11] Demographic and health indicators for the Eastern Mediterranean 2006. Cairo, WHO Regional Office for the Eastern Mediterranean, 2006.

[12] Ngayawanaka, I. P. Influence of maternal socio-economic status on neonatal mortality trends, by region, Cameroon, 2012.

[13] Akoto, M. E. Infant mortality in Africa: level and characteristics, causes and determinants, CIACO, 1985.

[14] Barry, B. Maternal Mortality in Bakel Department: Causes and Favourable Factors Determined by the Verbal Autopsy, Master's thesis on Research, 2008.

[15] WHO, Maternal Mortality: Removing women from the deadly spiral. WHO Chronicle, 40 (5), page 193-202, 1986.

[16] Dicko-Traoré, F., Sylla, M., Traoré, Y., Traoré, A., Diall, H., Diakité. National reference neonatology unit in Mali: state of play. Public Health 2014; 26 (1), 115-121.

[17] Katamea T, Mukuku O, Kamona L, Mukelenge K, Mbula O, Baledi L, et al. Mortality risk factors in newborns transferred to the neonatal unit at Jason Sendwe Hospital in Lubumbashi, Democratic Republic of Congo. Pan African Medical Journal. 2014; 19: 169. 